\title{
GROWN: Local Data Compression in Real-Time To Support Energy Efficiency in WBAN
}

\author{
Cainã Passos*, Carlos Pedroso*, Agnaldo Batista*, Michele Nogueira*, Aldri Santos* \\ ${ }^{*}$ Wireless and Advanced Networks Laboratory (NR2) - UFPR, Brazil \\ Emails:\{cpassos, capjunior, asbatista, michele, aldri\}@inf.ufpr.br
}

\begin{abstract}
The evolution of wireless technologies has enabled the creation of networks for several purposes as health care monitoring. The Wireless Body Area Networks (WBANs) enable continuous and real-time monitoring of physiological signals, but that monitoring leads to an excessive data transmission usage, and drastically affects the power consumption of the devices. Although there are approaches for reducing energy consumption, many of them do not consider information redundancy to reduce the power consumption. This paper proposes a hybrid approach of local data compression, called GROWN, to decrease information redundancy during data transmission and reduce the energy consumption. Our approach combines local data compression methods found in WSN. We have evaluated GROWN by experimentation, and the results show a decrease in energy consumption of the devices and an increase in network lifetime.
\end{abstract}

\section{INTRODUCTION}

Wireless sensors networks (WSN) and nanosystems have enabled the deployment of wearables sensors added into clothing or implanted in the body, such as fitness trackers and smartwatches [1]. Wireless body area networks (WBANs) enables the collect, monitoring, and transmission of physiological signals from people to several medical applications and health professionals, making patients day-to-day easier [2] [3]. The WBAN devices usually employ small batteries, which limit the energy consumption to communication [2]. Further, wearable and implantable devices working on realtime should have a long lifetime, mainly due to challenges for battery recharge and/or replacement [4]. Thus, manners for saving transmission energy can support the services provided, increasing the network lifetime. Although works have proposed, for instance, communication protocols to maximize the network lifetime, they did not focus on the redundancy of sensed data, transmitting all the collected information.

Data compression techniques by local data lossy or lossless allow us to aggregate and decrease the gathered information redundancy in WBAN [5]. While in lossy compression, we discard an acceptable amount of information, reducing the number of transmitted bits and increasing data compression rates, in lossless compression, the information remains unchanged [6]. Some works have employed lossy compression by a threshold between each transmitted data [7], [8], while others employ codification tables, decreasing the amount of transmitted data and preserving their integrity [9], [10]. However, as WBAN sensors collect distinct kinds of data, they require both techniques to work in a hybrid way [5], [11]. Therefore, the management of the compression service must be adaptive to the characteristics of the detected data to be energy efficient. Though many of works that take into account signal heterogeneity disregard the maximum latency set for WBAN to medical applications, $125 \mathrm{~ms}$, and to other ones, 250ms [2].

This work presents a mechanism for the management of local data compression in real-time and energy-efficient to WBAN called GROWN (EnerGy-Efficient Local Data Compression for TRansmission Over WBAN). GROWN coordinates the compression to the type of the collected data signal, avoiding redundant data transmission and thus reducing devices' energy consumption. GROWN applies lossy compression by a threshold to limit the acceptable data losses during transmissions, and lossless compression by encoding tables, which normalizes the data format to decrease the amount of transmitted information. Experimentation points out that GROWN increases the lifetime of sensors up to $53.73 \%$ with a maximum delay of $55 \mathrm{~ms}$ between consecutive data samples, showing gains to WBAN working in real-time.

The paper is organized as follows: Section II shows the related works. Section III describes the GROWN system and its operation on the WBAN. Section IV shows an experimental evaluation and the results obtained. Section $\mathrm{V}$ presents the conclusion and future works.

\section{RELATED WORK}

The literature has shown various local and hybrid data compression techniques [5], [8]-[11]. The local data compression classes take advantages, such as reduced information redundancy and lower power consumption in wireless transmission. However, compressing data can lead to increased latency in the delivery of information, in some cases, higher energy consumption is due to the need for more computational processing for compression. Among works that have applied local data compression for WBANs, in [8], an amendment to the traditional lightweight temporal compression (LTC) method has been proposed by [12] to minimize the error rate of information rebuilding and decrease the redundancy of the information transmitted. They also added the differential pulse coding modulation (DPCM) method into the compression phase to define these combinations according to the target application. While both methods increase the compression rates and decrease the number of transmissions, the developed method needs the storage of the data set to be transmitted over a time of 60 seconds to check the consecutive samples; this issue directly impact on information delay, usually a primary 
requirement for WBAN [2]. In [9], a lossless method explores the correlation between consecutive samples to get an efficient table of dynamics for Huffman coding. Though, the method exhibited high processing rates due to the constant updates of the dynamic table, affecting the device's energy consumption. In [10], the authors presented a lossless compression method based on the discrete wavelet transform (DWT) as using a lifting scheme [13]. Thus, the transformation of redundant samples in a given period of time can be compressed with a smaller number of bits. Although the method minimized the redundancy of the information and achieved high compression rates, its computational cost requires an excessive energy consumption from the devices. The use of the local data compression classes separately offers improvements to the efficiency of the body devices. In [5], DPCM was applied for loss compression and the coding tables for Exponential of Golomb (Exp-Golomb) [14] with changes for lossless compression. The method achieved high compression rates, however, the energy consumption of the devices and the compression delay is not explained. In [11], a hybrid-search method decreases the power consumption of devices and improves the quality of the electrocardiogram (ECG) signal. The data are compressed with lossy compression, suitable for the preliminary evaluation of ECG signals; and in cases that require more detailed analysis, it uses lossless data compression. Although there is a considerable reduction of the power consumption of the devices, the method only classified one type of signal and disregarded information such as device latency and memory.

\section{REAL-TIme LOCAL DATA COMPRESSION}

This section presents an overview of the collect and dissemination data environment, the GROWN components and their operation functioning. GROWN decreases the amount of transmitted information from wearable devices (e.g., sensors) to the central entity (e.g., sink), whose processing and energy resources allow operation while not affecting the latency in WBAN. We also assume that the sink device disseminates sensitive data collected from body devices to outside devices to enable them to follow data evolution over time.

GROWN carries out on a set of wearable portable devices (nodes) for sensing personal physiological signals interconnected in a WBAN, whose devices are denoted by $D=\left\{d_{1}, d_{2}, d_{3}, \ldots, d_{j}\right\}$, where $d_{j} \in D$, as shows Fig. 1

These devices possess computational resources to collect and disseminate physiological signals. All devices keep a unique identifier $(I d)$ to identify it over time and perform the

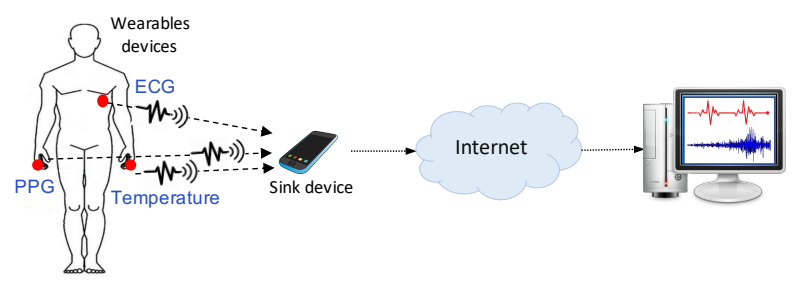

Fig. 1: Gather and dissemination model of signals tasks of signal gather and processing, as well as sending the information to the sink device. By simplicity, we assume that the wireless communication technology controls the message losses between sensors and the sink device. Also, nodes operate statically over time in a star network topology.

The GROWN architecture takes the Compression Management (CM) and Decompression Management (DM) modules, as shown in Fig. 2. The former makes the compression of the physiological signals received from wearable devices and the latter decompresses and recovers the original information collected from sensors. We detail each one as follows.

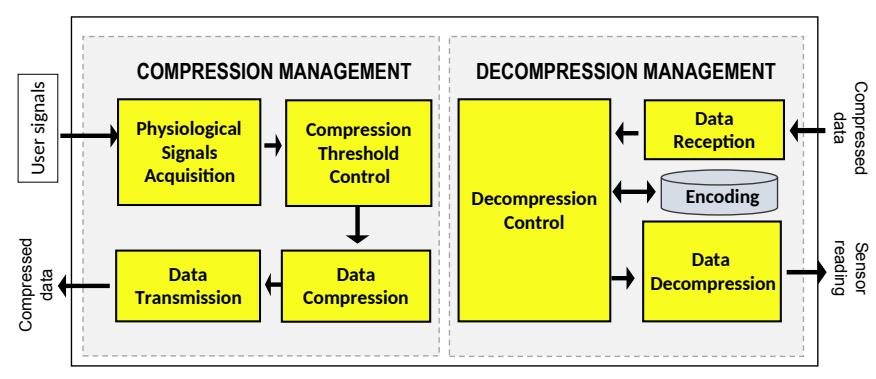

Fig. 2: The GROWN architecture

\section{A. Data Compression Module}

The CM module consists of components that convert the physiological signal from analog to digital, analyze the digital data and define the form of compression, perform data compression and transmit that data to the sink device through a wireless connection for further decompression. Wearable devices collect physiological signals from the user through the Physiological Signals Acquisition (PSA) component. This component converts the analog physiological signal to digital and sends it to the Compression Threshold Control (CTC) component. As described in Algorithm 11, this component receives the current reading from the sensor $(l .1)$. The first reading of the sensor (l.3) is then stored for comparison with the next ones (l.4), and also sent for compression (l.6.14). In the next readings, if the variation between the current and the previous reading exceeds the previously established threshold (l.8-9), the difference is forwarded to compression (l.11) to decrease the amount of transmitted information, and the last reading stored for the next comparison (l.12).

GROWN compresses the information $\left(e_{t s}\right)$ received by $C T C$ using a modified version of the Exponential-Golomb code (Exp-Golomb) of order 3 [14]. Thus, each received sample $\left(e_{t s} \neq 0\right)$ is represented as a bit sequence $\left(b s_{i}\right)$ composed of two parts $s_{i} \mid a_{i}$. The first part $\left(s_{i}\right)$ identifies the group to which $e_{t s}$ belongs and illustrates the value of $n_{i}$, which is equivalent to the number of bits needed to represent $e_{t s}$. Thus, we represent the groups $s_{i}$ related to the first $2^{k}-1$ values of $n_{i}$ by $\left\lfloor\log _{2}\left(\left|e_{t s}\right|\right)\right\rfloor+1$, where $s_{i}$ is represented by $k$ bits. When $n_{i}>6$, the value of the $s_{i}$ group is determined by $\left\lfloor\log _{2}\left(\left|e_{t s}\right|\right)-1\right\rfloor$, where the first $s_{i}-1$ values of $s_{i}$ are represented by 1 followed by 0 (zero). The $a_{i}$ part corresponds 


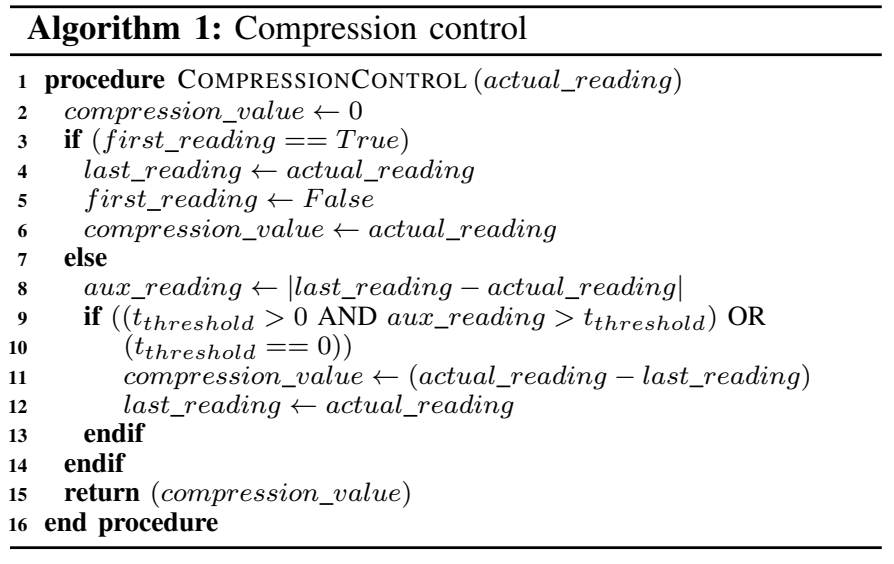

to the binary representation of $e_{t s}$, whose variable-length code is defined according to [15], ensuring different values for the entries of each group. Table I shows the rules for $e_{t s}$ values.

TABLE I: Data compression rules

\begin{tabular}{l|l}
\hline Condition & Rule \\
\hline$e_{t s}<0$ & $\begin{array}{l}\text { Make a } 2 \text { complement of } e_{t s}, \text { subtract } 1 \text { and use } \\
\text { the least significant } n_{i} \text { bits }\end{array}$ \\
$e_{t s}=0$ & $\begin{array}{l}\text { Encode } s_{i} \text { as } 000 \text { and not represent } a_{i} \\
e_{t s}>0\end{array}$ \\
& $\begin{array}{l}a_{i} \text { corresponds to the less significant } n_{i} \text { bits of } \\
\text { the } e_{t s} 2 \text { complement }\end{array}$ \\
\hline
\end{tabular}

Table II shows the number of $n_{i}$ bits for representing $e_{t s}$ and the encoding values for each group $s_{i}$. Thereby, enconding depends on differences between the values of $e_{t s}$, thus more frequent differences imply shorter code. After $e_{t s}$ compression, the new information $\left(\operatorname{In} f_{t s}\right)$ is transmitted to the sink device.

TABLE II: Encoding values

\begin{tabular}{l|rrrc}
\hline$n_{i}$ & \multicolumn{1}{|r}{$s_{i}$} & \multicolumn{1}{c}{$e_{t s}$} & bit & byte \\
\hline 0 & 000 & 0 & 3 & 1 \\
1 & 001 & $-1,+1$ & 4 & 1 \\
2 & 010 & $-3,-2,+2,+3$ & 5 & 1 \\
3 & 011 & $-15, \ldots,-8,+8, \ldots,+15$ & 7 & 1 \\
4 & 100 & $-31, \ldots,-16,+16, \ldots,+31$ & 8 & 1 \\
5 & 101 & $-63, \ldots,-32,+32, \ldots,+63$ & 9 & 2 \\
6 & 110 & $-127, \ldots,-64,+64, \ldots,+127$ & 12 & 2 \\
7 & 11110 & $-255, \ldots,-128,+128, \ldots,+255$ & 14 & 2 \\
8 & 111110 & & \\
9 & 1111110 & $-511, \ldots,-256,+256, \ldots,+511$ & 16 & 2 \\
\hline
\end{tabular}

\section{B. Decompression management module}

This module plays in the sink device, which has enough resources of power, storage, and processing to carry out the decompression of data sent by wearable devices in the WBAN. Thus, as depicted in Fig. 2, the recovery of each physiological signal starts in the Data Reception component. This component receives the message with the compressed data $\left(b s_{i}\right)$ and the device identification $\left(D_{i d}\right)$, and forwards it to the Decompression Control (DC) component, according to Algorithm 2. DC holds a reference list (RL) to store the identification of all wearable devices in the WBAN, besides

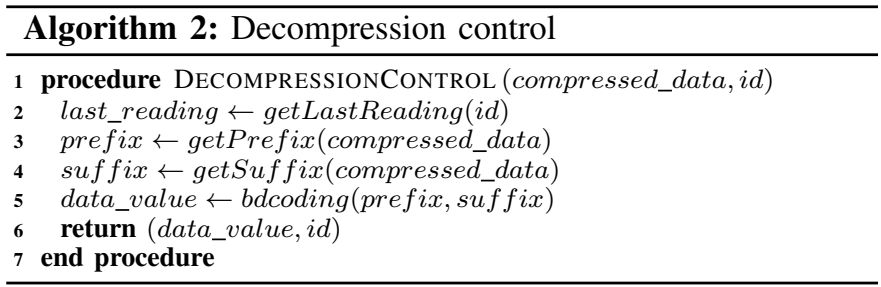

the values of their last reading $\left(\hat{x}_{t s}\right)(l .2)$. Next, CD obtains the prediction error $\left(e_{t s}\right)$ through the prefix $s_{i}(l .3)$ and the suffix $a_{i}(l .4)$ from $b s_{i}$. Then, it queries the Encoding database (l.5), which stores the values of indexes $\left(n_{i}\right)$, groups $\left(s_{i}\right)$ and the binary representation of $e_{t s}\left(a_{i}\right)$. As $a_{i}$ is unique in $s_{i}$, the recovery of the received $b s_{i}$ occurs with few instructions, adapting to the real-time operation. Thus, DC forwards $\hat{x}_{t s}$ and $e_{t s}$ values to the Data Decompression (DD) component and updates the RL. Lastly, DD decompress the data and makes it available to others applications.

\section{Operation}

Fig. 3 depicts a wearable device in a WBAN continuously sensing a person's temperature, meanwhile it interacts with the sink device (e.g., smartphone). The sensor starts collecting the temperature signal and converts it from analog to digital in a binary representation $\left(x_{t s}\right)$ with $R$ bits. Suppose its reading is 38 , so $x_{t s}$ value in 8 bits is 001001100 . As the decisionmaking about the data compression relies on a pre-defined prediction filter $T$, assume $T=1$. Hence, the filter checks the $x_{t s}$ value and as this is the first reading, the last reading $\left(\hat{x}_{t s}\right)$ gets the value 0 . Thus, the prediction error $\left(e_{t s}\right)$, which means the difference between consecutive readings, is equal to $e_{t s}=x_{t s}-\hat{x}_{t s}=38$.

Next, the $e_{t s}$ value is encoded to a bit sequence $\left(b s_{i}\right)$ in two parts, $s_{i} \mid a_{i} . s_{i}$ represents the group $\left(n_{i}\right)$ which $e_{t s}$ belongs, so $n_{i}=\left\lfloor\log _{2}(|38|)\right\rfloor+1=6$. By the encoding values in Table [I] we verify that the $s_{i}$ value belongs to the group 110 . The $a_{i}$ value comes from the conversion of the $e_{t s}=38$ value to a binary form, $a_{i}=100110$, so $b s_{i}=110100110$. After that, the wearable device sends a message to the sink device carrying $b s_{i}$ and $D_{I d}$ values. When the sink receives this message, it recovers the $s_{i}$ and $a_{i}$ values from $b s_{i}$ and gets the prediction error $e_{t s}$ by querying the encoding table using $b s_{i}$ and $D_{I d}$ values. Through RL, it obtains $\hat{x}_{t s}$ from $e_{t s}$ and $D_{I d}$. As it is the first reading, $\hat{x}_{t s}=0$. Finally, it retrieves the information equivalent to the reading of the wearable device, In $f_{t s}=e_{t s}+\hat{x}_{t s}=38$.

\section{Evaluation}

This section describes the GROWN implementation and analyzes how the management of lossless (LL) and lossy (LS) compression provides energy efficiency to WBAN. GROWN combines distinct platforms to meet the needs of wearable and sink devices to mimics a real environment in experimentation. We have compiled the compression module in $\mathrm{C}++$ language in Arduino boards, whose integrated microcontroller operates 


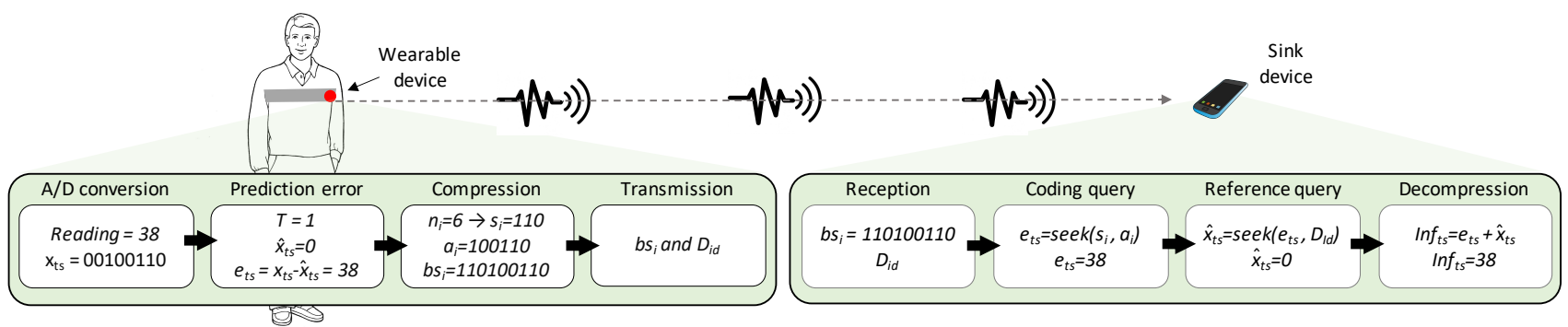

Fig. 3: The step by step of GROWN operation

with a $16 \mathrm{MHz}$ clock and a 10 bits resolution in each logical port for physiological sensors readings. We have developed the decompression module in Android Studio, version 3.6.1, that was installed in the sink device, a Motorola smartphone, model $\mathrm{G}^{4}$ Plus, with an Android version 7.0.

We built a testbed composed by three wearable devices to act as physiological sensors and one smartphone to operate as the WBAN's sink device, as depicted in Fig. 1 . We set up the wearables devices on protoboards model Mb102 to sense temperature, ECG and photoplethysmogram (PPG) signals, as described in Table III. These protoboards provide energy to sensors and Arduino boards supplied by $5 \mathrm{~V}$ regulated power sources supported by $9 \mathrm{~V} / 400 \mathrm{mAh}$ batteries. Each wearable device has a sensor to collect a given physiological signal and all the sensors codes were compiled with official libraries.

To enhance the GROWN analysis in the ECG signals compression, we have added a 4th device (ECGDB) to our testbed that acts as an ECG sensor and employs the MIT-BIH arrythmia database [16] as its data source. The wearables and sink devices communicate by Bluetooth Low Energy (BLE), by IEEE 802.15.1 standard, through a HM-10 BLE V4 module. They keep a connection during 600s, a sufficient period to get 600 instantaneous current reading samples from each device. We set up the smartphone to operate in flight mode during the experiments to avoid any interruptions from other applications, while keeping in operation only the Bluetooth communication. As shown in Fig. 4, the environment for sensing a person's body temperature operates by infrared signals to avoid the physical contact of the person's body with the device. After the reading, the device compresses such data and forwards to the Bluetooth module that transmits it to the sink device, which then decompresses the data. We have set up the prediction filter to $1(\mathrm{~T}=1)$ for lossy compression to improve the identification of the correlation between consecutive samples.

TABLE III: Wearable devices settings

\begin{tabular}{l||ccc}
\hline \multirow{2}{*}{ Feature } & \multicolumn{3}{|c}{ Device } \\
\cline { 2 - 4 } & Temperature & PPG & ECG / ECGDB \\
\hline Board & Arduino UNO & Arduino UNO & Arduino Mega \\
Controller & ATmega328P & ATmega328P & ATmega2560 \\
Body sensor & MLX90614 & PulseSensor & AD8232 \\
\hline
\end{tabular}

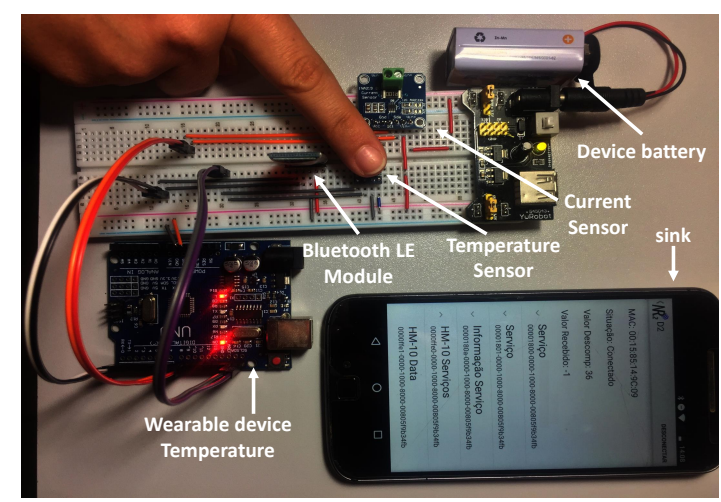

Fig. 4: Wearable temperature device testbed

The analysis of GROWN takes into account the metrics presented in Table IV] We have measured the Compression Delay $(C D)$ and Decompression Delay $(D D)$ by [17], and the Average Delay $(A D)$ by [18]. We have also employed a current and voltage sensor from Texas Instruments, model INA219 [7] to measure the Device Energy Consumption $(D E C)$. This sensor monitors the voltage drop over a $1 \mathrm{~m} \Omega$ shunt resistor, which is proportional to the current flowing through it [19]. We obtained the Compression Ratio (PCR) from the amount of compressed packets (comp_pkt) and the amount of these packets without any compression (orig_pkt). Results exhibited correspond to an average of ten repetitions.

TABLE IV: Evaluation Metrics

\begin{tabular}{l||l}
\hline Metric & Equation \\
\hline Compression Delay (CD) & $\sum_{i=1}^{n} \frac{T_{f c o m} i-T_{i c o m} i}{I n f_{t s} i}$ \\
\hline Decompression Delay (DD) & $\sum_{i=1}^{n} \frac{T_{f d e s} i-T_{i c d e s} i}{I n f_{t s} i}$ \\
\hline Average Delay (AD) & $\sum_{x=1}^{N_{e}} \sum_{y=1}^{T_{t r}} \frac{C D_{x y}+D D_{x y}+D T R_{x y}}{T_{t r} \times N_{e}}$ \\
\hline Energy consumption (DEC) & $I \times t$ \\
\hline Compression Ratio (PCR) & $\left(1-\frac{c o m p \_p k t}{o r i g_{-} p k t}\right) \times 100$ \\
\hline
\end{tabular}

\section{A. Results}

The compression and decompression processes add a delay to data dissemination, which varies according to the correlation between consecutive samples. The temperature signals exhibit 

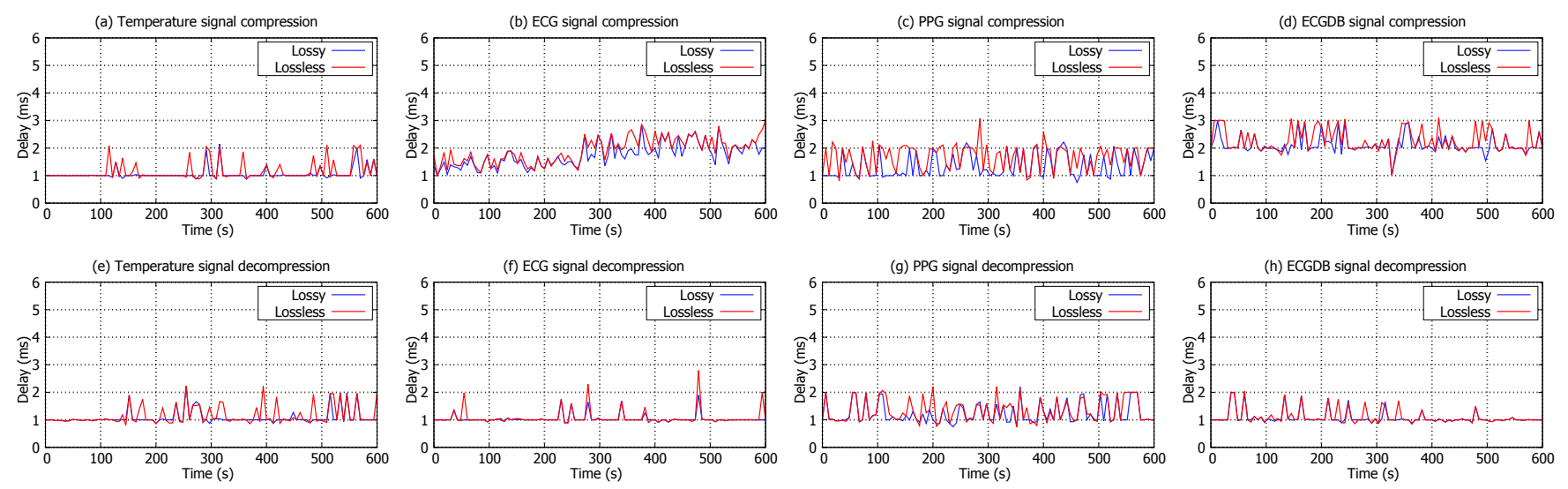

Fig. 5: Delays in compression and decompression of physiological signals

high correlated samples, whose consecutive values present slight variations. Hence, GROWN measured a value of $\approx 1 \mathrm{~ms}$ to $\mathrm{CD}$, the lowest among four types of signals (Fig. 5(a)). We noted that the samples correlation of ECG (Fig. 5. (b)) and PPG (Fig. 5. (c)) signals frequently varies over time, so both suffered higher $\mathrm{CD}, \approx 3 \mathrm{~ms}$ and $\approx 2 \mathrm{~ms}$, respectively. Finally, as shows Fig. 5[d), ECGDB signals behaved in similar way and cost $\approx 3 \mathrm{~ms}$ for CD. On the other hand, as depict Fig. 5.e), 5 (f), $5 \mathrm{~g}$ ), and $5(\mathrm{~h})$, the GROWN cost was $\approx 1 \mathrm{~ms}$ for DD, as the smartphone that plays as sink device has greater computational resources than wearables devices.

We have identified that signals with low correlated consecutive samples (i.e., consecutive values exhibit wide variations) increase the compression time in devices with constrained resources. Fig. 6 shows the Average Delay (AD) measured on the evaluated physiological signals with lossless (LL) and lossy (LS) compression. We noted that ECG, ECGDB and PPG signals show $\approx 3 \mathrm{~ms}$ of $\mathrm{AD}$, while the temperature signal shows $\approx 1 \mathrm{~ms}$. However, the delay of $\approx 49 \mathrm{~ms}$ on all wearable devices is due to the manner Bluetooth BLE connections happen, endorsing what Gatouillat et al. in [20] have already identified on Bluetooth connections on Android 7.0. In a general manner GROWN raised $7.84 \%$ on the total transmission time for ECG and ECGDB signals, 6\% for PPG, and $2.04 \%$ for temperature signal. Those values show GROWN's ability in saving energy on data dissemination in WBANs, which demand a maximum latency of $125 \mathrm{~ms}$ for medical applications and 250ms for non-medical ones [2].

We also noted that consecutive highly correlated samples enable devices to reduce energy consumption (DEC), as shows Fig. 7. because GROWN assigns shorter codes for the small differences to be transmitted. To verify its energy efficiency, we have conducted experiments with lossless (CGLL) and lossy (CGLS) compression, and also without compression (CGWC). We verified that the temperature device consumes on average $36.81 \mathrm{mAh}$ for CGLL and $36.65 \mathrm{mAh}$ for CGLS, while in CGWC it consumes $38.28 \mathrm{mAh}$. Hence, GROWN achieves an energy efficiency of $1.47 \mathrm{mAh}$ for CGLL and $1.63 \mathrm{mAh}$ for CGLS. GROWN reached an energy efficiency

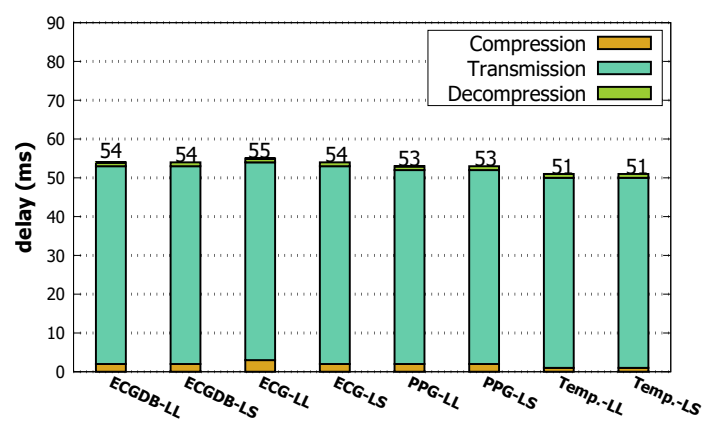

Fig. 6: Delay in processing physiological signals

of $1.02 \mathrm{mAh}$ on the ECG device for both methods. For the PPG device, we observe an energy efficiency of $0.31 \mathrm{mAh}$ for CGLL and $0.89 \mathrm{mAh}$ for CGLS, as this kind of signal presents low correlated consecutive samples. As the ECGDB device has performed frequent readings of ECG samples from the MITBIH database in the SD module, this approach has increased its energy consumption to $73.29 \mathrm{mAh}$ in CGWC, and produces an energy efficiency of $0.90 \mathrm{mAh}$ for CGLL and $1.14 \mathrm{mAh}$ for CGLS. Although data compression also consumes energy, even so there is an increase of the lifetime of each wearable device with the use of GROWN, as we can see in Table $\mathrm{V}$

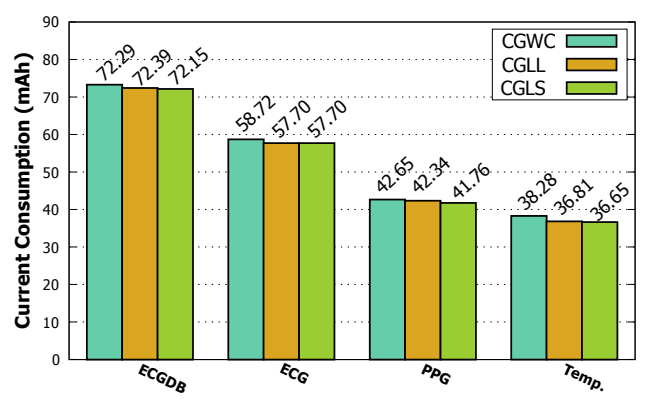

Fig. 7: Energy consumption of wearables devices

GROWN enables an energy efficiency to the devices even with their Bluetooth module active and paired with the 
TABLE V: Lifetime of wearable devices

\begin{tabular}{l|l||cccc|c}
\hline \multicolumn{2}{l||}{ Mode } & \multicolumn{4}{|c|}{ Normal } & Sleep \\
\hline \multicolumn{1}{l|}{ Device } & ECGBD & ECG & PPG & Temperature & Temperature \\
\hline \multirow{3}{*}{ Battery life $(h)$} & CGWC & 5.46 & 6.81 & 8.38 & 10.45 & - \\
& CGLL & 5.53 & 6.93 & 9.45 & 10.87 & 15.50 \\
& CGLS & 5.54 & 6.93 & 9.58 & 10.91 & 16.05 \\
\hline
\end{tabular}

smartphone. But, only this condition already increases the energy consumption, as this module carries a CC2540 BLE chip, which consumes $24 \mathrm{~mA}$ to data transmission and reception [21]. Hence, to reduce energy consumption, we have set up the Bluetooth module in sleep mode. So, it falls asleep after a certain period and wakes up with a proper command. We have set up the temperature device to this configuration, as it has the highest correlated consecutive samples and it is active whenever the device does not transmit two consecutive samples. This strategy has reduced DEC for the temperature device to $25.81 \mathrm{mAh}$ for CGLL and to $24.92 \mathrm{mAh}$ for CGLS, both from CGWC. Hence, as shows Table V, it has increased temperature device lifetime by $53.73 \%$ for CGLL, from $10.45 \mathrm{~h}$ in normal mode to $15.50 \mathrm{~h}$ in sleep mode, and by $48.37 \%$ for CGLS, from $10.45 \mathrm{~h}$ in normal mode to $16.05 \mathrm{~h}$ in sleep mode.

TABLE VI: Compression ratio of physiological signals

\begin{tabular}{l||cccccc}
\hline \multirow{2}{*}{ Signal } & \multicolumn{2}{c}{ Num. of delivered packets } & & \multicolumn{2}{c}{ Compression ratio (\%) } \\
\cline { 2 - 4 } & Original & LS & LL & & LS & LL \\
\hline ECGDB & 8.863 & 5.589 & 5.757 & & 36.94 & 35.04 \\
ECG & 7.586 & 4.879 & 4.879 & & 36.68 & 36.68 \\
PPG & 6.483 & 5.011 & 6.016 & & 22.70 & 7.20 \\
Temp. & 120 & 1 & 2 & 99.16 & 97.50 \\
\hline
\end{tabular}

GROWN got higher data compression ratio (PCR) mainly for highly correlated samples, as shows Table VI. We noted that it greatly decreases the number of transmitted packets, and reduces the energy consumption. On the temperature signal, GROWN achieved a PCR of $99.16 \%$ for LS, and $97.50 \%$ for LL, as its high correlated consecutive samples. Meanwhile, on the ECG signal, it got a PCR of $35.68 \%$ for both methods, as the correlation between consecutive samples exceeded the threshold of 1 , and obliges the transmission of the same amount of information. As this behavior occurs less frequently with PPG signal, GROWN achieved the lowest PCR on it.

\section{CONCLUSiON}

This paper presented GROWN, a mechanism to save energy and increase the lifetime of wearable devices in wireless body networks. It manages real-time data compression in the sensing device by techniques employed in WSNs, so it allows devices to achieve an energy efficient operation. Experiments evaluated the GROWN effectiveness, and the results have demonstrated its ability to manage data compression and decompression in real-time in WBAN. GROWN has achieved an energy efficiency up to $53.73 \%$ with a maximum delay of $55 \mathrm{~ms}$. Future works we will investigate GROWN availability in the face of data losses in the transmission, and its reliability with other wireless communication technologies. We also intend to compare GROWN with other mechanisms.

\section{ACKNOWLEDGMENT}

We would like to acknowledge the support of the Brazilian Agency CNPq in the Universal Project, grant \#436649/2018-7.

\section{REFERENCES}

[1] T. Tavares, M. Nogueira, D. Rosário, A. Santos, and E. Cerqueira, "Traffic Model Based on Autoregression for PPG Signals in Wearable Networks," IEEE Networking Letters, vol. 2, no. 2, pp. 49-53, 2020.

[2] S. Movassaghi, M. Abolhasan, J. Lipman, D. Smith, and A. Jamalipour, "Wireless body area networks: A survey," IEEE Communications Surveys \& Tutorials, vol. 16, no. 3, pp. 1658-1686, 2014.

[3] P. Resque, S. Pinheiro, D. Rosário, E. Cerqueira, A. Vergutz, M. Nogueira, and A. Santos, "Assessing Data Traffic Classification to Priority Access for Wireless Healthcare Application," in IEEE LATINCOM 2019. IEEE, 2019, pp. 1-6.

[4] Y. Liao, M. Leeson, Q. Cai, Q. Ai, and Q. Liu, "Mutual-informationbased incremental relaying communications for wireless biomedical implant systems," Sensors, vol. 18, no. 2, p. 515, 2018.

[5] G. Giorgi, "A combined approach for real-time data compression in wireless body sensor networks," IEEE Sensors Journal, vol. 17, no. 18, pp. 6129-6135, 2017.

[6] T.-H. Tsai and W.-T. Kuo, "An Efficient ECG Lossless Compression System for Embedded Platforms With Telemedicine Applications," IEEE Access, vol. 6, pp. 42 207-42 215, 2018.

[7] F. Marcelloni and M. Vecchio, "Enabling energy-efficient and lossyaware data compression in wireless sensor networks by multi-objective evolutionary optimization," Information Sciences, vol. 180, no. 10, pp. 1924-1941, 2010.

[8] J. Azar, A. Makhoul, R. Darazi, J. Demerjian, and R. Couturier, "On the performance of resource-aware compression techniques for vital signs data in wireless body sensor networks," in IEEE Middle East and North Africa Communications (MENACOMM). IEEE, 2018, pp. 1-6.

[9] C. P. Antonopoulos and N. S. Voros, "Resource efficient data compression algorithms for demanding, wsn based biomedical applications," Journal of biomedical informatics, vol. 59, pp. 1-14, 2016.

[10] J. Azar, R. Darazi, C. Habib, A. Makhoul, and J. Demerjian, "Using dwt lifting scheme for lossless data compression in wireless body sensor networks," in 14th Int. Wireless Communications \& Mobile Computing Conference (IWCMC). IEEE, 2018, pp. 1465-1470.

[11] C. J. Deepu, C.-H. Heng, and Y. Lian, "A hybrid data compression scheme for power reduction in wireless sensors for iot," IEEE trans. on biomedical circuits and systems, vol. 11, no. 2, pp. 245-254, 2017.

[12] T. Schoellhammer, B. Greenstein, E. Osterweil, M. Wimbrow, and D. Estrin, "Lightweight temporal compression of microclimate datasets [wireless sensor networks]," 29th Annual IEEE International Conference on Local Computer Networks, pp. 516-524, Nov 2004.

[13] W. Sweldens, "The lifting scheme: A construction of second generation wavelets," SIAM journal on mathematical analysis, vol. 29, no. 2, pp. 511-546, 1998.

[14] J. Teuhola, "A compression method for clustered bit-vectors," Information processing letters, vol. 7, no. 6, pp. 308-311, 1978.

[15] F. Marcelloni and M. Vecchio, "A simple algorithm for data compression in wireless sensor networks," IEEE comm. letters, vol. 12, no. 6, 2008.

[16] G. B. Moody and R. G. Mark, "MIT-BIH Arrhythmia Database," https://physionet.org/content/mitdb/1.0.0/, 2005, [Online]. Acessed in Jan. 2020.

[17] D. Patel, V. Bhogan, and A. Janson, "Simulation and comparison of various lossless data compression techniques based on compression ratio and processing delay," IJCA, vol. 81, no. 14, 2013.

[18] D. Zordan, B. Martinez, I. Vilajosana, and M. Rossi, "On the performance of lossy compression schemes for energy constrained sensor networking," ACM TOSN, vol. 11, no. 1, pp. 1-34, 2014.

[19] J. E. Parks, "Ohms Law III Resistors in Series and Parallel," Department of Physics and Anatomy, University of Tennessee, 2007.

[20] A. Gatouillat, B. Massot, Y. Badr, E. Sejdić, and C. Gehin, "Building iot-enabled wearable medical devices: an application to a wearable, multiparametric, cardiorespiratory sensor," 2018.

[21] T. Instruments, "CC2540 Bluetooth® Low Energy wireless MCU with USB," https://www.ti.com/product/CC2540. 2013, (accessed March 1, 2020). 